Moral Sense und praktische Vernunft 


\title{
Moral Sense und \\ praktische Vernunft
}

David Humes Ethik und Rechtsphilosophie

\author{
von \\ Bernd Gräfrath
}

J.B.Metzlersche Verlagsbuchhandlung Stuttgart 
CIP-Titelaufnahme der Deutschen Bibliothek

Gräfrath, Bernd:

Moral Sense und praktische Vernunft : David Humes Ethik und Rechtsphilosophie / von Bernd Gräfrath. - Stuttgart : Metzler, 1991

ISBN 978-3-476-00742-1

ISBN 978-3-476-03347-5 (eBook)

DOI 10.1007/978-3-476-03347-5

Dieses Werk einschließlich aller seiner Teile ist urheberrechtlich geschützt. Jede Verwertung außerhalb der engen Grenzen des Urheberrechtsgesetzes ist ohne Zustimmung des Verlages unzulässig und strafbar. Das gilt insbesondere für Vervielfältigungen. Übersetzungen, Mikroverfilmungen und die Einspeicherung und Verarbeitung in elektronischen Systemen.

(C) 1991 Springer-Verlag GmbH Deutschland

Ursprünglich erschienen bei J.B. Metzlersche Verlagsbuchhandlung und Carl Ernst Poeschel Verlag GmbH in Stuttgart 1991 
I come now to the last Charge, which, according to the prevalent Opinion of Philosophers in this Age, will certainly be regarded as the severest, viz. the Author's destroying all the Foundations of Morality.

He hath indeed denied the eternal Difference of Right and Wrong in the Sense in which Clark and Wollaston maintained them, viz. That the Propositions of Morality were of the same Nature with the Truths of Mathematicks and the abstract Sciences, the Objects merely of Reason, not the Feelings of our internal Tastes and Sentiments. In this Opinion he concurs with all the antient Moralists, as well as with Mr. Hutchison Professor of Moral Philosophy in the University of Glasgow, who, with others, has revived the antient Philosophy in this Particular. How poor the Artifice, to cite a broken Passage of a Philosophical Discourse, in order to throw an Odium on the Author! 


\section{VORWORT}

David Hume nimmt in der Tradition der anglo-amerikanischen Philosophie eine ähnlich bedeutsame Stelle ein wie Immanuel Kant in der kontinental-europäischen Tradition. Die vorliegende Arbeit ist getragen von der Absicht, zur Überwindung der Trennung in zwei verschiedene Traditionen, die einander nichts zu sagen haben, beizutragen. David Hume wird zwar inzwischen auch in Deutschland als Klassiker anerkannt, aber seine praktische Philosophie ist hierzulande bislang kaum rezipiert worden. Im folgenden soll durch einen frischen interpretatorischen Zugriff deutlich werden, daB Hume in seinem eigenen Werk einer Spaltung in zwei verschiedene Traditionen Empirismus und Rationalismus, Emotivismus und Kognitivismus - gerade entgegentreten will. Dies herauszuarbeiten ist nicht nur von historischem Interesse, sondern könnte darüber hinaus einen fruchtbaren Anstoß für die gegenwärtige systematische Diskussion bilden.

Auch das vergleichsweise abstrakte Gebiet der Metaethik hat dabei durchaus praktische Relevanz: Die Frage nach der »Subjektivität« oder »Objektivität« moralischer Werturteile hat Auswirkungen auf die Frage nach der Pflicht zum Rechtsgehorsam; und die Utilitarismus-Debatte hat Auswirkungen auf die Kontroverse über den moralischen Status von Tieren. Auch die Moral- und Rechtsphilosophie gehören zur praktischen Ethik.

Konstanz/Berlin, im Sommer 1990

Bernd Gräfrath 


\section{Inhalt}

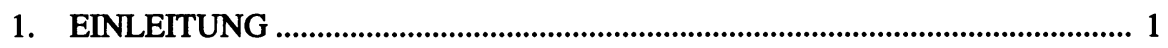

2. DIE METAETHIK DAVID HUMES ….................................................... 5

2.1 Hares Systematik metaethischer Theorien ..................................................... 5

2.2 Drei einseitige Fehldeutungen von Humes Metaethik ....................................... 7

2.2.1 Die emotivistische \$Standard «-Interpretation .................................... 7

2.2.2 Die subjektiv-naturalistische Interpretation ........................................ 9

2.2.3 Die »objektivistische «Interpretation ............................................... 12

2.3 Humes Metaethik im historischen und systematischen Zusammenhang.... 15

2.3.1 Die Theorie des moral sense ...................................................... 15

2.3.1.1 Broads Systematik von moral sense-Theorien .................... 15

2.3.1.2 Shaftesbury ……................................................................ 17

2.3.1.3 Hutcheson ..................................................................... 19

2.3.2 Hume über sekundäre Qualitäten und das Geschmacksurteil in der Ästhetik ............................................................................ 22

2.4 Humes metaethische Theorie .................................................................... 29

2.4.1 Grundlegende Aspekte von Humes Metaethik ................................. 29

2.4.1.1 Die Rolle von reason und sentiment in der moralischen Urteilsbildung .................................................................. 29

2.4.1.2 Das ethische Sprachspiel und der Standpunkt der Moral 33

2.4.1.3 Die menschliche Natur und das Problem des Relativismus ...................................................................... 37

2.4.2 Zusammenfassung der metaethischen Theorie Humes ..................... 39

2.5 Zur Aktualität von Humes Metaethik ........................................................... 42

3. DIE NORMATIVE ETHIK DAVID HUMES …………..................................... 45

3.1 Deontologische und teleologische Theorien ................................................. 45

3.2 Der Regelutilitarismus .............................................................................. 46

3.3 Drei einseitige Interpretationen von Humes normativer Ethik .................... 49 
3.3.1 Die regelutilitaristische $»$ Standard «-Interpretation ......................... 49

3.3.2 Die gesellschaftsvertragliche Interpretation .................................. 53

3.3.3 Die aristotelische Interpretation .................................................... 59

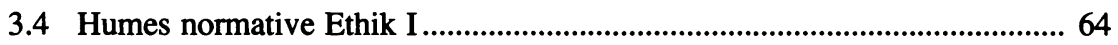

3.4.1 Humes Staatstheorie: Soziale Regelsysteme als Produkte des kollektiven Egoismus ....................................................................... 64

3.4.2 Das Trittbrettfahrerproblem ........................................................ 72

3.4.3 Egoismus, Sympathie und moralisches Urteil ................................ 80

3.5 Humes Rechtstheorie: Rechtspositivismus oder Naturrechtslehre? ............. 86

3.5.1 Rechtspositivismus und Naturrechtslehre ......................................... 86

3.5.2 Zwei Interpretationen von Humes Rechtslehre ............................... 94

3.5.2.1 Die rechtspositivistische »Standard «-Interpretation ............ 94

3.5.2.2 Die naturrechtliche Interpretation ...................................... 96

3.5.3 Exkurs: Hume - ein Konservativer!? ............................................ 98

3.6 Humes normative Ethik II: Zur Bedeutung von Humes Theorie natürlicher Tugenden ...................................................................... 103

4. DETERMINISMUS, FREIHEIT UND VERANTWORTLICHKEIT .............. 113

4.1 Hume über den Determinismus und seine Folgen .................................. 113

4.2 Humes »weicher« Determinismus und die strafrechtliche Theorie von der »Charakterschuld « ............................................................ 120

4.3 Windelbands Determinismus-Kritik und die Möglichkeit einer kompatibilistischen Theorie in der Tradition Humes ................................ 128

5. HUMES BESTIMMUNG DES ADÄQUATEN AUFGABENBEREICHS DES RECHTS

5.1 Rechtsliberalismus, Rechtspaternalismus und Rechtsmoralismus ............ 135

5.2 Rechtsliberalistische und rechtspaternalistische Konzepte bei Hume ....... 142

6. ZUSAMMENFASSUNG: VIERMAL RECHT UND MORAL ....................... 146

7. ANHANG I: HUME ZUM MORALISCHEN UND RECHTLICHEN STATUS DER TIERE

7.1 Hume zum moralischen Status von Tieren ............................................. 149

7.2 Zum rechtlichen Status von Tieren ...................................................... 156 


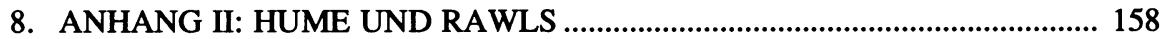

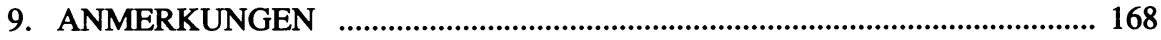

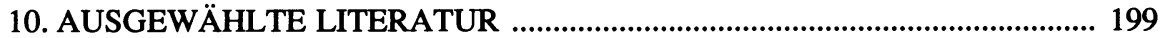

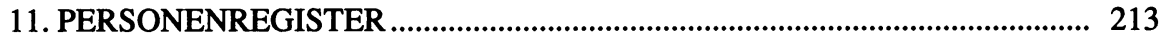

